\title{
Studying frequency processing of the brain to enhance long-term memory and develop a human brain protocol
}

\author{
Wernher Friedrich ${ }^{\mathrm{a}}$, Shengzhi Du ${ }^{\mathrm{b}, *}$ and Karlien Balt ${ }^{\mathrm{c}}$ \\ ${ }^{a}$ School of Computing, University of South Africa, Pretoria, South Africa \\ ${ }^{\mathrm{b}}$ The Department of Mechanical Engineering, Tshwane University of Technology, Pretoria, South \\ Africa \\ ${ }^{\mathrm{c}}$ Neurofeedback, Lynnwood, Pretoria, South Africa
}

\begin{abstract}
.
BACKGROUND: The temporal lobe in conjunction with the hippocampus is responsible for memory processing. The gamma wave is involved with this process. To develop a human brain protocol, a better understanding of the relationship between gamma and long-term memory is vital.

OBJECTIVE: A more comprehensive understanding of the human brain and specific analogue waves it uses will support the development of a human brain protocol.

METHODS: Fifty-eight participants aged between 6 and 60 years participated in long-term memory experiments. It is envisaged that the brain could be stimulated through binaural beats (sound frequency) at $40 \mathrm{~Hz}$ (gamma) to enhance long-term memory capacity. EEG recordings have been transformed to sound and then to an information standard, namely ASCII.

RESULTS: Statistical analysis showed a proportional relationship between long-term memory and gamma activity. Results from EEG recordings indicate a pattern. The pattern was obtained through the de-codification of an EEG recording to sound and then to ASCII.

CONCLUSIONS: Stimulation of gamma should enhance long term memory capacity. More research is required to unlock the human brains' protocol key. This key will enable the processing of information directly to and from human memory via gamma, the hippocampus and the temporal lobe.
\end{abstract}

Keywords: Electroencephalography (EEG), signal processing, brain protocol, long-term memory, hippocampus

\section{Introduction}

Electroencephalography (EEG) has been used in many domains in order to study and better understand the human brain.

EEG signals have been classified in specific categories such as alpha [8-13 Hz], beta [13-22 Hz], delta $[0.5-4 \mathrm{~Hz}]$, theta $[4-8 \mathrm{~Hz}]$ and gamma [22-30 Hz] to name a few frequency ranges [112]. It should be noted that these categories are in proximity and do vary slightly.

In recent years focus has been placed on P300, which can be classified as a type of event related potential (ERP). The output from P300 occurrences enable researchers to study human brain activity

\footnotetext{
${ }^{*}$ Corresponding author: Shengzhi Du, The Department of Mechanical Engineering, Tshwane University of Technology, Pretoria, South Africa. E-mail: dus@tut.ac.za. 
based on stimuli received and are usually observed between $250-500 \mathrm{~ms}$ after stimulus [3]. P300 is a method being used in the area of lie-detection [4], and other research areas including brain-computer interfacing (BCI) [5].

Various research methods have been applied to EEG signals in order to extract and study specific features, one of these is a link that gamma has with long-term memory.

In this research ERPs such as P300 support futuristic research, whereby a human brain protocol could be developed for communication and memory purposes. EEG experimentation and results obtained relating to gamma wave activity and long-term memory have proved that a direct link exists. Further research into the stimulation of gamma waves will be focused on using sound frequency differing in pitch by $40 \mathrm{~Hz}$. The experiment to stimulate gamma wave activity will be done to determine if weak long-term memory capacity can be increased through the stimulation of the gamma wave. The reason why long-term memory capacity is critical, is because facts that are required to be stored for long periods of time, reside within long-term memory. If a person has weak long-term memory, using a brain protocol to store and read information from such individuals could be compromised, due to the fact that the brain protocol will process and use gamma waves for reading and storing of information in the brain's natural form via gamma waves. Low gamma wave activity could result in the inability to use gamma as a path to communicate with the brain's natural memory processing capabilities. Binaural beats [6] are the method to be used where a sound frequency differing by $40 \mathrm{~Hz}$ is presented to each ear of a participant. Further research is to be conducted in the use of sound and conversion techniques for the en- and de-codification of information within specific brain waves such as gamma. The en- and de-codification of information is still at an experimental stage and this paper will focus on the progress achieved thus far as well as laying the foundation for future work to be done.

\section{Methods}

\subsection{Method used for gamma recording}

EEG data from a group of 58 people was analyzed. The group consisted of 30 males and 28 females between the ages of 6-60 years. The database of patients enabled a wide sample to be referenced in order to determine if there is a relationship between gamma and long-term memory capacity. Recordings of data followed the 10/20 system and were performed by a specialist in neurophysiology. The device used for data acquisition was a Brainmaster Atlantis II $4 \times 4$ with MiniQ II. Individuals were fitted with an ECI (Electro Cap International) cap and normal conductive ECI Electro Gel (for use with non-disposable neuro-diagnostic surface electrodes during EEG exams and brain mapping) was applied within electrodes via a syringe to obtain EEG signals [7]. Impedances were checked to ensure they remained below $5 \mathrm{k}$ Ohms.

Data was recorded over five minutes. The EEG-machine records data for 4 channels per minute, recording a total of 20 channels over 5 minutes. The procedure was then repeated with the individual sitting with their eyes closed. Data was then analyzed using Neuro-guide software which is able to combine data into one recording.

\subsection{Method used for brain protocol recording}

An alternative EEG recording was used to determine the close relationship that exists between EEG signals and existing standardized en-codification protocols, such as ASCII (American Standard Code for Information Interchange). This recording was performed on a g.tec gUSBamp [9] following the 10/20 system (Fig. 1). 16 electrodes were used connected to the scalp via a cap and band pass filtering was 


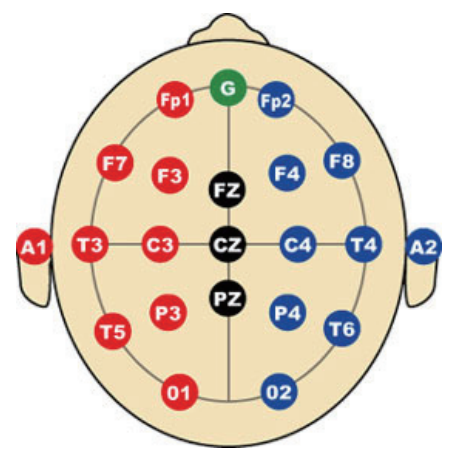

Fig. 1. International 10/20 system [8], Standard placements of electrodes for EEG recordings.

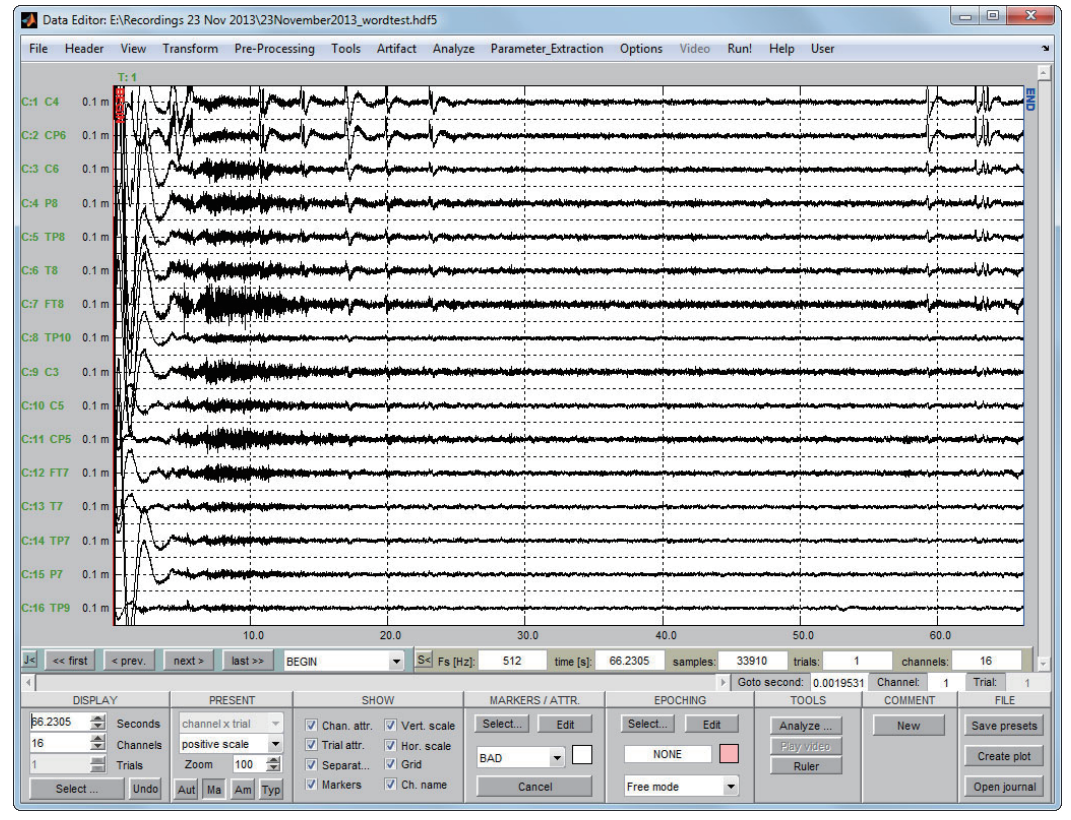

Fig. 2. g.tec EEG data viewer, EEG recording displaying electrical brain activity.

set to $0.5-60 \mathrm{~Hz}$, notch filtering was set at $50 \mathrm{~Hz}$. Recordings were done in a controlled laboratory environment, using a projector to display words and images to participants. Participants were selected that did not have any known memory problems and had good overall memory capabilities. Long-term memory was gauged to be at an acceptable level if a participant could recall events from their childhood. Images, words and a story were selected that supported recognition and required long-term memory processing and recall. Content selected formed part of general public knowledge as well as general knowledge for participants such as flowers, animals and cars. The story selected was unique but the language was known.

Figure 2 shows the recordings of 16 channels. Data was then analyzed via Matlab and g. tec analysis tools. The frequency spectrum of the recordings is shown in Fig. 3. Figure 4 illustrates the conversion from EEG data to sound. Conversion was then taken one step further, from sound to binary in order to obtain an ASCII character encoding representation.

Figure 3 illustrates EEG signal converted from time domain to frequency domain via the FFT algorithm. Different frequencies can be observed such as alpha, beta, delta and gamma, as well as the percentage of a specific frequency that forms part of the overall signal. FFT is being used to determine what frequencies are present inside an EEG signal.

Figure 4 illustrates an EEG signal that has been converted to sound. Very high and low digital voltages recorded during EEG are being displayed as high and low amplitudes.

\section{Results}

\subsection{Gamma results}

Results from the group of 58 individuals indicated that there is a direct relationship between gamma wave activity and long-term memory capacity. 
Table 1

Summary of long-term memory capacity and gamma activity

\begin{tabular}{llll}
\hline Groups & \multicolumn{1}{c}{ Hits } & \multicolumn{1}{c}{ Mis-hits } & Uncertain \\
\hline Weak LTM & $54.5 \%(6$ of 11$)$ & $18.2 \%(2$ of 11$)$ & $27.3 \%(3$ of 11$)$ \\
Normal LTM & $70.2 \%(33$ of 47$)$ & $4.3 \%(2$ of 47$)$ & $25.5 \%(12$ of 47$)$ \\
\hline
\end{tabular}

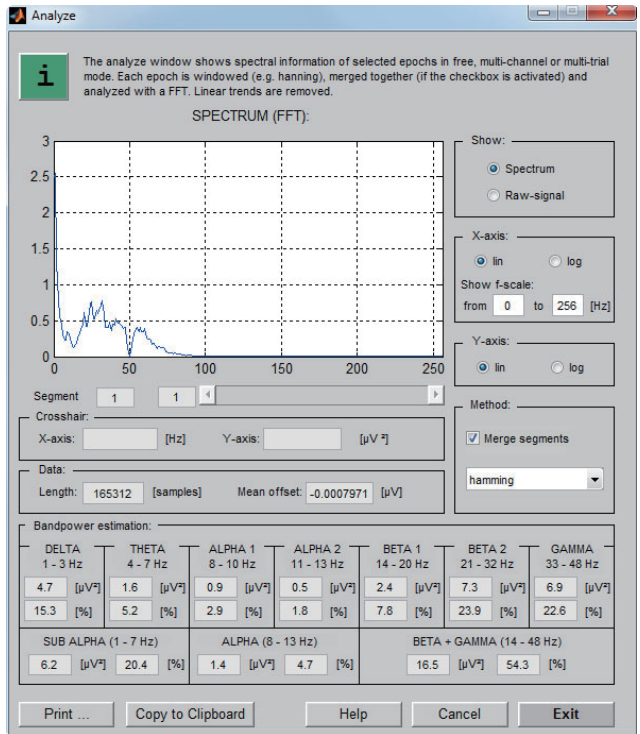

Fig. 3. g.tec FFT analyzer.

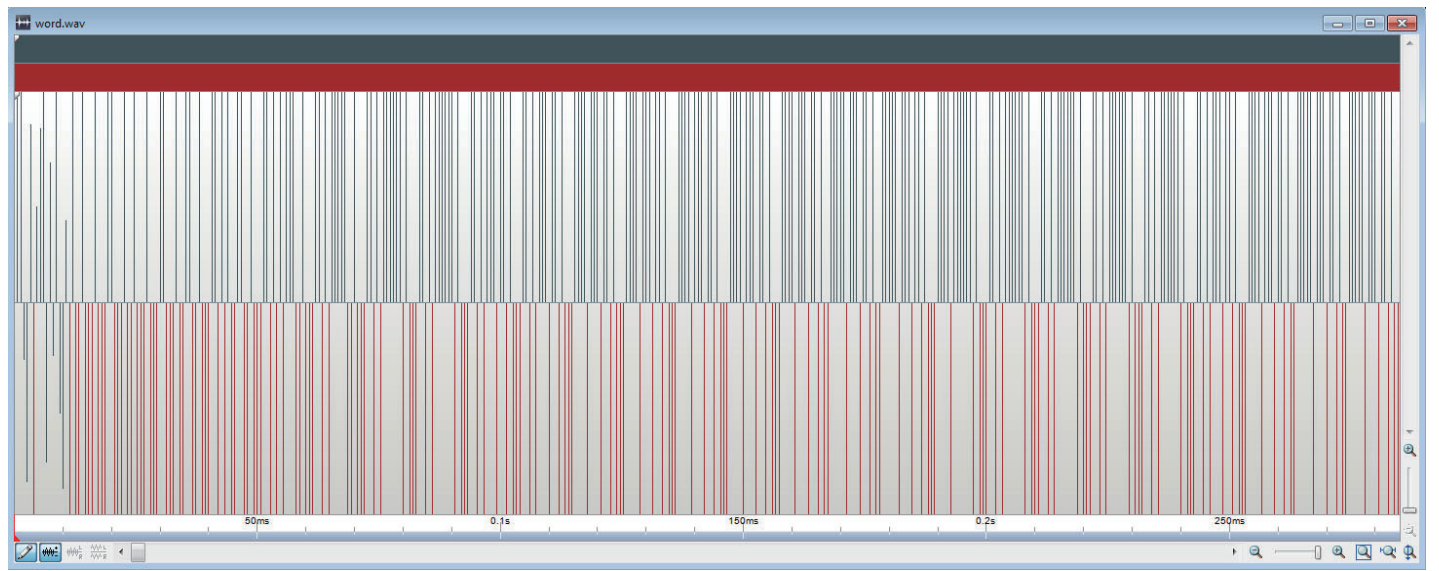

Fig. 4. Converted EEG recording to sound.

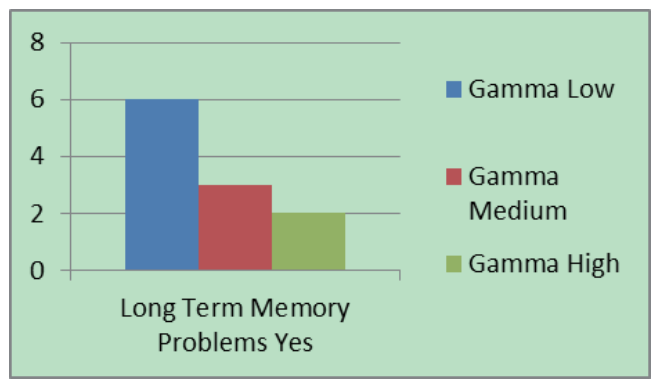

Fig. 5. Eleven testers with weak long-term memory.

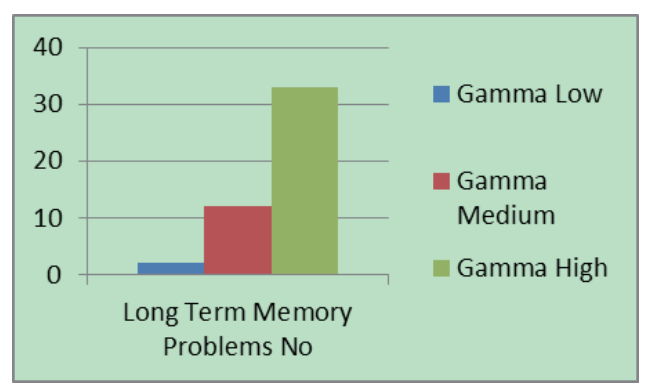

Fig. 6. Forty-seven testers with normal long-term memory. 


\section{ASCII Code: Character to Binary}

\begin{tabular}{|c|c|c|c|c|c|c|c|}
\hline 0011 & 0000 & 0 & 0100 & 1111 & m & 0110 & 1101 \\
\hline 0011 & 0001 & $P$ & 0101 & 0000 & n & 0110 & 1110 \\
\hline 0011 & 0010 & $Q$ & 0101 & 0001 & $\circ$ & 0110 & 1111 \\
\hline 0011 & 0011 & $\mathbf{R}$ & 0101 & 0010 & p & 0111 & 0000 \\
\hline 0011 & 0100 & $\mathbf{s}$ & 0101 & 0011 & a & 0111 & 0001 \\
\hline 0011 & 0101 & $\mathbf{T}$ & 0101 & 0100 & $r$ & 0111 & 0010 \\
\hline 0011 & 0110 & U & 0101 & 0101 & $\mathbf{s}$ & 0111 & 0011 \\
\hline 0011 & 0111 & $\mathrm{v}$ & 0101 & 0110 & $t$ & 0111 & 0100 \\
\hline 0011 & 1000 & w & 0101 & 0111 & u & 0111 & 0101 \\
\hline 0011 & 1001 & $\mathrm{x}$ & 0101 & 1000 & $\mathrm{v}$ & 0111 & 0110 \\
\hline 0100 & 0001 & $X$ & 0101 & 1001 & $\mathrm{w}$ & 0111 & 0111 \\
\hline 0100 & 0010 & z & 0101 & 1010 & $\mathbf{x}$ & 0111 & 1000 \\
\hline 0100 & 0011 & a & 0110 & 0001 & $y$ & 0111 & 1001 \\
\hline 0100 & 0100 & b & 0110 & 0010 & $z$ & 0111 & 1010 \\
\hline 0100 & 0101 & c & 0110 & 0011 & - & 0010 & 1110 \\
\hline 0100 & 0110 & d & 0110 & 0100 & , & 0010 & 0111 \\
\hline 0100 & 0111 & e & 0110 & 0101 & : & 0011 & 1010 \\
\hline 0100 & 1000 & f & 0110 & 0110 & ; & 0011 & 1011 \\
\hline 0100 & 1001 & g & 0110 & 0111 & $?$ & 0011 & 1111 \\
\hline 0100 & 1010 & h & 0110 & 1000 & I & 0010 & 0001 \\
\hline 0100 & 1011 & I & 0110 & 1001 & ' & 0010 & 1100 \\
\hline 0100 & 1100 & $j$ & 0110 & 1010 & " & 0010 & 0010 \\
\hline 0100 & 1101 & $\mathrm{k}$ & 0110 & 1011 & 1 & 0010 & 1000 \\
\hline \multirow[t]{2}{*}{0100} & 1110 & 1 & 0110 & 1100 & 1 & 0010 & 1001 \\
\hline & & & & & pac & 0010 & 0000 \\
\hline
\end{tabular}

Fig. 7. Example of the ASCII en-codification table [10].

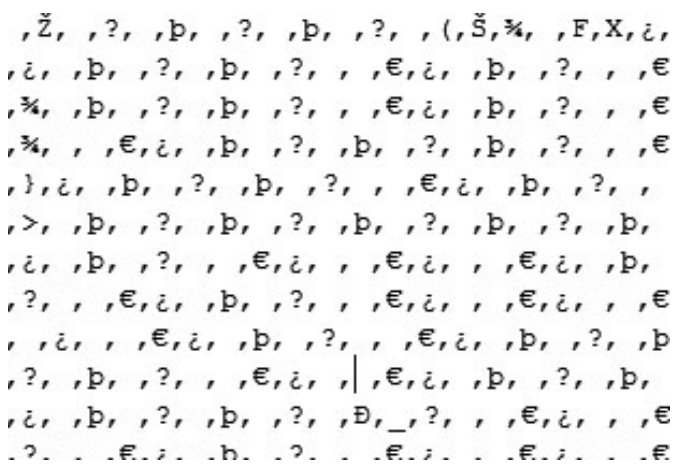

Fig. 8. Pattern result from EEG conversion to ASCII.

Figures 5 and 6 indicate the statistics with regards to the gamma wave activity levels of weak and strong long-term memory groups from the total of 58 individuals. The data is summarized in Table 1.

In Table 1, the term LTM represents long-term memory. In the row of "Weak LTM"; the column "Hits" refers to persons with weak long-term memory and low-gamma activity. However, the "Mis-hits" in the same row refers to weak long-term memory persons that have high-gamma activity which does not support the result. In the row of "Normal LTM"; "Hits" means normal LTM and high-gamma while "Mis-hits" means normal LTM but low-gamma activity. The "Uncertain column" represents persons having a medium gamma level.

The conclusion that can be drawn from the data is that gamma activity is strongly proportional relevant to the long-term memory capacity: $67.2 \%$ (39 of 58) cases support the relationship with only $6.9 \%$ (four of 58) negative cases.

This conclusion will be further tested in future research where sound frequency with a difference of $40 \mathrm{~Hz}$ in pitch will be used. Positive results would mean that specific frequencies can be stimulated in order to increase their resonating activity; with gamma this should increase long-term memory capacity.

\subsection{Brain protocol development results}

A very interesting result was obtained when EEG data was being analyzed for protocol capabilities. From a standardized point of view, an existing specific protocol to communicate directly with the human brain has not been developed yet. This paper makes an initial attempt at the development of such a protocol. The following steps were performed in the attempt.

(1) Conversion techniques to be able to get to a binary level. EEG data was converted from analogue to digital via sampling algorithms in order to be able to reconstruct the original signal as close 
as possible through digital values. The higher the sampling rate the better the quality of signal reproduction. In this research, EEG data was recorded at $512 \mathrm{~Hz}$ sampling; with band pass filtering of 0.5 to $60 \mathrm{~Hz}$. Notch filter was set at $50 \mathrm{~Hz}$ to remove electrical noise on recording;

(2) Convert digital values into sound (Fig. 4). This was done in order to enable digital values that are very high and low to be converted into high and low amplitudes;

(3) Conversion was done from sound into binary. The conversion process from sound enabled high and low amplitudes to be converted into $1 \mathrm{~s}$ and $0 \mathrm{~s}$;

(4) Binary was then converted to ASCII, to determine how close human brain frequency relates to an established en-codification protocol (Fig. 7).

The result in Fig. 7 shows an emerging pattern which indicates that the development of a brain protocol is a possibility.

The ASCII en-codification scheme only created a file that looks like an encrypted file, but the pattern that emerged indicated that if the correct en-codification scheme is developed, that information being processed and used inside the human brain can then be translated into human readable form.

\section{Discussion and conclusion}

The human brain has the capability to connect left and right brain lobes [6] when there is a difference in sound being experienced by a person. Binaural beats have been used to instigate sound differences in other research areas and will be used as a tool to enhance long-term memory capacity through gamma stimulation. Due to the nature of sound that exists in the frequency domain, a sound will be played that differs by $40 \mathrm{~Hz}$ (the frequency that gamma resonates on). It is envisaged that the brain should be able to recognize the difference and create a connection of $40 \mathrm{~Hz}$, which is the frequency targeted for gamma stimulation. If results prove positive, meaning gamma stimulation enhances a person's longterm memory capacity; the next step would be to stimulate other frequencies directly through sound. The authors acknowledge that while the experiment is largely theoretical, results will be analyzed and published.

Further research will also be done with regards to the protocol for human brain communication and memory formation. Currently the encoding schemes that exist do not follow the correct key to unlock the processing and recall of information inside the human brain. It is envisaged that with more EEG analysis and tests a key could be developed that can unlock human brain frequency en- and de-coding similar in nature as was attempted by [11], where P300 character en-coding and de-coding tests were conducted in order to reach character identification faster. The key would then support research in an attempt to establish a binary protocol for human brain frequency en-codification. The human brain protocol will then open the door for further research and understanding into communication and information processing inside the human brain. The research already conducted only lays the foundation for future research, but the benefits can truly be realized when implemented. Benefits will be realized where individuals are diagnosed with Alzheimer's, supporting memory preservation. Other areas include lie-detection [4] such as in the case of P300 and a deeper understanding in research areas such as EEG in attention deficit hyperactivity disorder [12], due to the nature of such brains to process information. Psychological benefits include support in overriding bad associations in trauma patients and possibly enhancing more positive, healthy associations in support of the healing process.

All benefits and research is only in its infancy, but the technological and en-codification protocol enhancement enables research into this domain. Acknowledgment is given that research is very experimental, such as the research currently being conducted by [13]14] where mice memory is being 
controlled and tested by way of electrodes invasively connected to the hippocampus in areas CA1 and CA3. Memory of mice has been switched on and off via electronic circuitry that either allows or disallows electrical signals to pass through to the area responsible for memory processing and formation. EEG is non-invasive, but comparing mice with human brain activity is possible due to the fact that brains operate on a similar electrical and frequency basis.

\section{Acknowledgement}

Dr. Marina vd Ryst (Educational Psychologist), Forensic expert with special interest in Neuropsychology, is highly acknowledged for her dedicated input and continued support.

\section{References}

[1] The Basics of Brain Waves. Shue K. Brain and Health; 2014. Available from http://www.brainandhealth.com/BrainWaves.html.

[2] Transcendental Meditation. Myth \#2: Meditation and relaxation practices are basically all the same and produce the same effects; 2014. Available from http://meditationasheville.blogspot.com/2010/12/myth-meditation-and-relaxation.html.

[3] P300 Neuroscience. 2014. Available from http://en.wikipedia.org/wiki/P300_\%28neuroscience\%29.

[4] Yijun X, Yu L, Wentao H, et al. A novel classification method based on ICA and ELM: a case study in lie detection. Bio-Medical Materials and Engineering 24. 2014; 357:363.

[5] Hoffmann U, Vesin J, Ebrahimi T, et al. An efficient P300-based brain - computer interface for disabled subjects. Journal of Neuroscience Methods Volume 167 Issue 1. 15 January 2008; 115:125.

[6] Binaural Beats. 2014. Available from http://en.wikipedia.org/wiki/Binaural_beats.

[7] Brainmasters Inc. 2014. http://www.brainmaster.com/content-sub/atlantis-ii/.

[8] Immrama Institute. The International 10-20 System of Electrode Placement; 2014. Available from http://immrama.org/ $? \mathrm{~s}=10-20$

[9] gtec Medical Engineering; 2014. Available from http://www.gtec.at/Products/Hardware-and-Accessories/g.USBampSpecs-Features.

[10] Basic Binary Code. 2015 [cited 2015 Jan 18]. Available from http://www3.amherst.edu/ jcook15/binarycode.html.

[11] Fukami T, Shimada T, Forney E, et al. EEG character identification using stimulus sequences designed to maximize minimal hamming distance. 2012 IEEE Annual International Conference of the Engineering in Medicine and Biology Society (EMBC).

[12] Liu T, Lin P, Chen Y, et al. Electroencephalogram synchronization analysis for attention deficit hyperactivity disorder children. Bio-Medical Materials and Engineering 24. 2014; 1035:1039.

[13] Chan RHM, et al. Changes of Hippocampal CA3-CA1 Population Nonlinear Dynamics across Different Training Sessions in Rats Performing a Memory-Dependent Task. 32nd Annual International Conference of the IEEE EMBS Buenos Aires Argentina. August 31 - September 42010.

[14] Popular Science. Boyle R; 2011 [cited 2011 June 18]. http://www.popsci.com/technology/article/2011-06/artificialmemory-chip-rats-can-remember-and-forget-touch-button. 